\title{
Carcinoma de células de Merkel: patogénesis, manejo y tratamientos emergentes
}

\author{
Merkel Cell Carcinoma: Pathogenesis, Management and Emerging Treatments
}

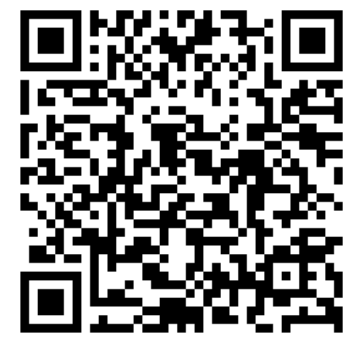

${ }^{1}$ Médico general, graduado de la Universidad de Ciencias Médicas (UCIMED).

daniel.barquero95@hotmail.com

${ }^{2}$ Médico especialista en Oncología Médica, graduado de la Universidad de Costa Rica (UCR). Código medico:6103 denislandaverde@gmail.com

${ }^{3}$ Médico general, graduado de la Universidad de Costa Rica (UCR).

segurajime01@gmail.com

\author{
${ }^{1}$ Dr. Daniel Esteban Barquero Orias \\ Investigador independiente, San José, Costa Rica \\ daniel.barquero95@hotmail.com \\ (iD https://orcid.org/0000-0001-7627-1358 \\ ${ }^{2}$ Dr. Denis Ulises Landaverde Recinos \\ Hospital Mexico, San José, Costa Rica \\ denislandaverde@gmail.com \\ https://orcid.org/0000-0002-6522-1061
}

${ }^{3}$ Dra. Jimena María Segura Guevara Investigadora independiente, San José, Costa Rica segurajime01@gmail.com

(D) https://orcid.org/0000-0002-0887-3717

RECIBIDO

$13 / 03 / 2019$
CORREGIDO

27/03/2019
ACEPTADO

09/04/2019

\section{RESUMEN}

El carcinoma de células de Merkel es un tumor raro neuroendocrino, altamente agresivo con altas tasas de mortalidad asociadas a sus metástasis a distancia. Es más frecuente en pacientes inmunosupresos, edad avanzada y exposición a luz ultravioleta. La mayoría de asocian con Poliomavirus sin embargo existen casos sin presencia viral. Se recomienda la resección quirúrgica con márgenes extensos cuando se encuentra localizado y en casos seleccionados la biopsia de ganglio centinela. Es una neoplasia quimiosensible y radiosensible. Existen avances terapéuticos inmunológicos para casos avanzados.

PALABRAS CLAVES: poliomavirus de células de Merkel; neoplasias cutáneas; radioterapia; inmunoterapia.

ABSTRACT
Merkel cell carcinoma is a rare neuroendocrine tumor, very aggressive
which has a high mortality rate when metastatic. It is more frequent in
immunocompromised as well as elderly patients and in those with
prolonged exposure to ultraviolet radiation. The Merkel Cell
polyomavirus has been found in the majority of the cases. Surgical
resection with or without sentinel lymph node biopsy with extensive


margins is recommended in early stages. It is a chemosensitive and radiosensitive neoplasm. There are immunological therapeutic advances for advanced cases.

KEYWORDS: Merkel cell polyomavirus; skin neoplasms; radiotherapy; immunotherapy.

\section{INTRODUCCIÓN}

Las células de Merkel fueron descritas inicialmente por Friedrich Sigmund Merkel, el cual las denomino "Touch Cells" debido su supuesto rol en las respuestas sensitiva-perceptiva táctil. Hoy en día tanto su origen como su función son inciertos $(1,2)$. Estas células residen de forma predominante en la capa basal de la epidermis y epitelio folicular donde se cree que están involucradas en síntesis de somatostatina, soporte neuronal y otros efectos paracrinos $y$ endocrinos (2). Estas células han demostrado receptores para percepción táctil y su inervación sensitiva es dada por nervios mielinizados $A \beta$ (3).

El carcinoma de células de Merkel (CCM) fue descrito en el año 1972 por Toker como un "carcinoma trabecular 'trabecular carcinoma' debido a la organización celular (4). Este tumor pertenece a la categoría de tumores de origen neural junto con el tumor neuroectodérmico (5). El CCM es conocido por diferentes nombres: carcinoma neuroendocrino primario de la piel, carcinoma cutáneo primario de células pequeñas y carcinoma trabecular cutáneo (3).

Es uno de los tumores cutáneos más agresivos y se ha reportado un aumento en su incidencia mundialmente (6), se localiza más frecuentemente a nivel de cabeza y cuello (7), con una frecuencia aumentada en pacientes con inmunocompromiso (5).

Existen casos reportados con regresión espontánea (8), sin embargo, el carcinoma se caracteriza por un curso agresivo con supervivencias a 10 años de $57.3 \%$ en Estados Unidos y $47 \%$ en Europa (9), aproximadamente un 30-40\% fallecerán a pesar del tratamiento a causa de su alto índice de metástasis a distancia (8). Esta revisión busca abordar las generalidades sobre este carcinoma como sus factores de riesgo, presentación clínica, abordaje y tanto tratamientos existentes como emergentes.

\section{EPIDEMIOLOGÍA}

La incidencia anual en Estados Unidos aumentó de 0,22 casos por 100000 habitantes en 1986 a 0,79 pacientes per 100000 habitantes en el 2011, se estima un número de 1600 casos anuales en Estados Unidos (4). El melanoma cutáneo es aproximamente 50 veces más común que el CCM (10). El CCM Predomina en población caucásica con menores tasas en pieles más pigmentadas como en poblaciones asiaticas o afroamericas (8). Pacientes infectados por el virus de inmunodeficiencia humano $(\mathrm{VIH})$ tienen aumento del riesgo relativo aproximadamente 13 veces más sobre pacientes no infectados y pacientes 
trasplantados tienen aumento de 10 veces más sobre la población general (3). La edad media al diagnóstico oscila entre 75-80 años (10), con mayor incidencia en el sexo masculino (11). Es más frecuente en zonas corporales fotoexpuestas como cabeza, cuello y extremidades donde se registran el $70-90 \%$ de los casos (1). En pacientes más jóvenes, es más común a nivel del tronco y en afroamericanos las extremidades inferiores son el sitio más común (2).

La mortalidad asociada se encuentra entre $33-46 \%$ a cinco años (12), siendo la segunda causa de mortalidad por cáncer cutáneo después del melanoma (11).

\section{ETIOLOGÍA Y PATOGÉNESIS}

El CCM es más frecuente en paciente con leucemia, linfoma o infección por VIH y en aquellos pacientes inmunosupresos por trasplante de órganos u otras causas (10), el $10 \%$ de los pacientes con esta entidad la constituyen aquellos con algún grado de inmunocompromiso y poseen peor pronóstico independiente al estadio comparado con los pacientes inmunocompetentes (8).

En un estudio de CCM asociado a trasplantes la incidencia aumentó tiempo después del trasplante sugeriendo un papel a largo plazo por la inmunosupresión crónica en lugar de la inmunosupresión aguda (13). Hasta el momento no hay estudios disponibles de manejo específico de la terapia inmunosupresora en receptores de trasplantes diagnosticados con CCM y la disminución de la dosis de la terapia inmunosupresorea debe ser individualizada (9). Los pacientes con carcinoma de células de Merkel tienen riesgo de malignidad secundaria como melanomas 0 malignidades hematológicas (14).

Se cree que a exposición solar es el factor de riesgo mayor para el desarrollo de este carcinoma (6). Se cree que la pigmentación cutánea tiene un efecto protector ya que pacientes asiáticos e hispanos tienen menor riesgo en comparación con individuos de piel blanca, se asocia a la exposición solar ya que es frecuente en pacientes de edad avanzada en piel con exposición solar crónica, aumento de incidencia en pacientes tratados con fototerapia y pacientes con historia de otras neoplasias cutáneas asociadas a exposición solar. Además, pacientes con historia de melanoma tiene riesgo triplicado de desarrollar CCM (10).

Algunos trastornos inflamatorios crónicos como la artritis reumatoide están asociadas con mayor incidencia en la aparición del carcinoma $(10,15)$.

Tumores en pacientes con trastornos inflamatorios crónicos expresaron niveles elevados del marcador de proliferación Ki67 comparado con grupo control, sugeriendo un curso más agresivo en pacientes con defectos en la inmunidad (15).

El poliomavirus de Celulas de Merkel (MCPyV, según sus siglas en inglés) se encuentra asociado en aproximadamente el $80 \%$ de los casos $(16,17)$, la infección primaria ocure durante la infancia y persiste latente en la piel por el resto de la vida (17). La prevalencia de infección subclínica aumenta con edad, estando presente en aproximadamente $60-80 \%$ de los adultos (18).

La presencia o no del virus asociado tiene variación geográfica siendo mayor en Estados Unidos y menor en países como Australia (19). Existe frecuente asociación 
de presencia de PCR positivo por poliomavirus en pacientes diagnosticados con enfermedades inflamatorias crónicas (15).

La proliferación de células con genes virales integrados sufren mutaciones que promueven la transformación (16). La vía oncogénica require integración al genoma celular antes de la proliferación tumoral (20). Los CCM revelan la expresión única de genes reguladores neuroendocrinos (REST, ASCL1, NeuroD1) asociados a un rol importante en la regulación celular (21). La evidencia sugiere la existencia de dos subgrupos (PCR positivo y PCR negativo por poliomavirus), con diferencias histopatológicas, inmunohistoquímicas, características oncogénicas y clínicas. Los tumores positivos por Poliomavirus son más frecuentes en el sexo femenino, localizados en extremidades, con estadios mas tempranos al momento de la presentación y con mejor supervivencia libre de enfermedad (20). La causa de tumores sin Poliomavirus es desconocida (1).

\section{PRESENTACIÓN CLÍNICA}

Su presentación mas común es un nódulo eritematoso con o sin superficie ulcerada usualmente $<2 \mathrm{~cm}$ de diámetro con predilección por zonas como cabeza y cuello en pacientes Caucásicos de edad avanzada. (1).

El carcinoma de células de Merkel es un tumor solitario de rápido crecimiento, asintomático, eritematoso o similar a la coloración cutánea, con características nodulares o en forma de placas. Se presenta en cabeza y cuello en un $53 \%$ de pacientes, extremidades en $34-35 \%$, tronco, mucosa oral y genital en menos del $10 \%(9)$. Se describe una entidad clínica a nivel de mucosas, con un comportamiento más agresivo (2).

El acrónimo "AEIOU" (A: Asintomático, E: Expansión rápido, I: Inmunosupresión, O: Older o Mayor a 50 años, U: Exposición a radiación UV) representa algunas características que hacen sospechar de carcinoma de células de Merkel, sin embargo, algunos tumores benignos cutáneos y otros tumores malignos pueden tener hasta 3 de estas características (22).

Aproximadamente $50-60 \%$ de pacientes se presentan con lesión primaria, sin embargo, se requiere valorar ganglios linfáticos ya que la incidencia de extensión subclínica es alta (30-50\%). Se han descrito algunos síndromes paraneoplásicos, usualmente asociados a otras malignidades neuroendocrinas así como en el carcinoma pulmonar de células pequeñas puede aparecer hiponatremia, degeneración cerebelosa y el síndrome de Lambert-Eaton (8).

\section{DIAGNÓSTICO DIFERENCIAL}

Se debe hacer diagnóstico diferencial de lesiones como queratoacantoma, queratosis seborreica, queratosis actínica, enfermedad de Bowen, carcinoma espinocelular, carcinoma basocelular variante esclerosante, pioderma gangrenoso, nevus, melanoma amelanótico, sarcoma de Kaposi, linfoma y angiosarcoma (5).

\section{PATOLOGÍA}

Bajo microscopía electrónica se han visualizado gránulos neuro-secretores (5). 
Presenta histología similar a tumores de células azules redondas pequeñas incluyendo carcinomas neuroendocrinos como el neuroblastoma, rabdomiosarcoma, carcinoide metastásico, , melanoma amelanocitico, condrosarcoma mesenquimatoso, sarcoma de Ewing, linfomas, osteosarcoma y carcinoma de células pequeñas de pulmón (11).

\section{- Inmunohistoquímica}

En la práctica clínica se recomienda complementar las características histológicas con inmunohistoquímica, se considera que el panel de marcadores debe incluir citoqueratina 20 (CK-20) y Factor-1 de Transcripción Nuclear Tiroideo (TTF-1) para establecer diagnóstico diferencial (9). El carcinoma de células de Merkel no se distingue morfológicamente del carcinoma de células pequeñas de pulmón metastásico (18), sin embargo, la mayoría de los carcinomas de células de Merkel son positivos para citoqueratina 20, sinaptofisina, cromogranina $A, y$ son negativos para citoqueratina 7 y TTF- 1 , siendo esto último importante para diferenciarlo del carcinoma de células pequeñas de pulmón metastásico (8).

\section{ESTADIAJE}

Imagenes radiológicas y la biopsia del ganglio centinela son útiles para determinar la extensión de enfermedad metastásica y valorar respuesta al tratamiento (12). La biopsia del ganglio centinela es una técnica efectiva y con mínima morbilidad para establecer compromiso micrometastásico de ganglios linfáticos con evidencia que en pacientes con biopsia negativa se puede omitir la linfadenectomía regional (8). Las micrometástasis en ganglios linfáticos se han detectado en alrededor del $30 \%$ de los casos según una revisión reciente de 721 casos, resaltando la utilidad de la biopsia de ganglio centinela en el manejo de esta patología(23).

Esta neoplasia puede crecer rápido y hacer metástasis en forma temprana con $63 \%$ de lesiones primarias con crecimiento rápido en los 3 meses previos al diagnóstico, $26 \%-36 \%$ con compromiso ganglionar y $6 \%-16 \%$ con metástasis distal al momento del diagnóstico (6).

\section{PRONÓSTICO}

La localización tumoral es un factor pronóstico ya que las neoplasias de cuero cabelludo se asocian más frecuentes con metastasis a distancia, los de oreja presentan la tasa más alta de metástasis ganglionar y los anogenitales tienen el peor pronóstico (4).

Neoplasias positivas por MCPyV tienen mejor pronóstico con mortalidad de $26 \%$ comparadas con $45 \%$ en pacientes con ausencia del virus $(8,16)$. El pronóstico clínico se ve afectado por factores como el sexo masculino, tamaño tumoral, edad avanzada, inmunosupresión y malignidades concomitantes. $(9,12)$. Individuos inmunosupresos tienen presentación más temprana y peor pronóstico comparados con grupo inmunocompetente (10).

Estudios han documentado recurrencia en $25 \%$ a $50 \%$ de los casos (6). Ya que casi todas las recurrencias ocurren en los primeros 2 años, con una media de recurrencia de 9 meses, se recomienda estudios radiológicos cada 3 a 6 meses en los primeros dos años (23). Se encuentra 
metastasis sin evidencia de primario en $10-20 \%$ de los casos, siendo éstos más difícil de manejar (22).

\section{TRATAMIENTO}

\section{- Cirugía}

Las guias del National Comprehensive Cancer Network (NCCN) recomiendan la resección quirúgica para obtener márgenes amplios de al menos 2-3 $\mathrm{cm}(22)$

Con cirugía convencional existe de $32 \%$ a $50 \%$ tasa de persistencia y recurrencia local. Varios estudios sugieren que la cirugía de Mohs ofrece ventajas en el tratamiento del este carcinoma sin embargo existen limitaciones por corto seguimiento y un pequeño número de individuos (24). Este procedimiento permite la conservación tisular y la identificación de tumores que requieren mayor disección (22), también permite el abordaje en zonas de cabeza y cuello donde en ocasiones no es posible una excisión amplia (8).

\section{- Radioterapia}

Se recomienda en casi todos los sujetos con tumor primario (7), es una neoplasia altamente radiosensible y la radiación de tumor primario y gánglios regionales se recomienda posterior a la escisión (24). No ofrece ventajas en presencia de ganglio centinela negativo (23), sin embargo, algunos autores recomiendan la radiación ganglionar en pacientes con ganglios patológicos negativos se debe individualizar, sin embargo se puede considerar en tumores con características de alto riesgo, $>2.0 \mathrm{~cm}$, invasión perineural o linfovascular y/o márgenes positivos (25). Es una opción razonable para pacientes en los cuales la cirugía no es una opción terapéutica (26).

\section{- Quimioterapia}

La quimioterapia se ha reservado principalmente para la enfermedad metastásica (19), actualmente ya no es la terapia estándar para el estadio IV (metástasis distales) debido al advenimiento de la terapia dirigida a los puntos de chequeo inmunológicos (6), sin embargo es razonable su uso cuando no es factible tener acceso a éstos fármacos o hay algún tipo de contraindicación a los mismos (14). Es una neoplasia quimiosensible con tasas de respuesta entre $53-61 \%$ con esquemas de primera línea basados en platino y $23-45 \%$ con esquemas de segunda línea (17). Como es un tumor neuroendocrino se utilizan esquemas similares a los utilizados para el carcinoma de células pequeñas de pulmón (5) uno de los regímenes preferidos es la combinación de agentes de platino y etopósido (27). Se recomienda uso de drogas combinadas y el uso de 5- fluoruracilo en pacientes que no toleran terapia por toxicidad o pacientes de edad avanzada (22). Supervivencia media con tratamiento es de alrededor de 9 meses (28).

\section{- Inmunoterapia}

Estudios clínicos de inmunofármacos sugieren que estas terapias podrían mejorar resultados clínicos desencadenando inmunidad antitumoral (27). Medicamentos nuevos incluyen el Avelumab, Pembrolizumab, Ipilimumab y Nivolumab (5).

Pembrolizumab fue el primer fármaco en demostrar regresión tumoral lo que llevo a su incorporación a las guías clínicas del National Comprehensive Cancer Network. 
Posteriormente se incluyó el Avelumab y el Nivolumab como parte del tratamiento preferido para enfermedad diseminada (27). Pembrolizumab fue efectivo tanto para neoplasias asociadas con virus como la no asociadas (29).

Avelumab reduce o elimina el efecto supresor del ligando 1 de muerte programada (PD-L1) sobre las células CD8+ lo que lleva a mejorar la respuesta anti-tumoral de las células T (17), sin embargo todavía no se ha determinado si la superioridad de este medicamento se debe a su doble capacidad de bloqueo de PD-L1 y promover la citotoxicidad celular dependiente de anticuerpos (30).

\section{CONCLUSIONES}

Se presenta más comúnmente como un nódulo eritematoso con o sin superficie ulcerada usualmente $<2 \mathrm{~cm}$ de diámetro, positivo a inmunotinción por citoqueratina 20 y negativos para TTF-1 estableciendo el diagnóstico diferencial con carcinoma de celulas pequeñas de pulmón.
Se destaca también la existencia de grupos con mayor riesgo como los pacientes con daño solar, edad avanzada, inmunosupresos, portadores de enfermedades inflamatorias crónicas y Caucásicos.

También, se asocia a la presencia de MCPyV en la mayoria de los casos. Estos pacientes tienen mayor riesgo de malignidad secundaria como melanomas y neoplasias hematológicos.

A partir de la revisión realizada se describe como un tumor agresivo, cuyo tratamiento principal en la enfermedad localizada es la resección quirúrgica con o sin radioterapia dependiendo de los factores de riesgo y estado ganglionar así como positividad de márgenes.

Se utiliza quimioterapia para los casos avanzados con metástasis distales, sin embargo no presenta duración en la respuesta, esto se ha modificado en los últimos años con el surgimiento de la inmunoterapia que han venido a ser de elección para tratar la enfermedad metastásica

\section{REFERENCIAS}

1. Brummer G, Bowen A, Bowen G. Merkel Cell Carcinoma: Current Issues Regarding Diagnosis, Management, and Emerging Treatment Strategies. American Journal of Clinical Dermatology. 2015;17(1):49-62. https://doi.org/10.1007/s40257-015-0163-3

2. Chteinberg E, Sauer C, Rennspiess D, Beumers L, Schiffelers L, Eben J et al. Neuroendocrine Key Regulator Gene Expression in Merkel Cell Carcinoma. Neoplasia. 2018;20(12):12271235.https://doi.org/10.1016/..neo.2018.10.003

3. Tsai S, Bordeaux J. Merkel Cell Carcinoma. A Practical Guide to Skin Cancer. 1 ed. Nashville: Springer; 2018. 143-153p.

4. Trinidad CM, Torres-Cabala CA, Prieto VG, Aung PP. Update on eighth edition American Joint Committee on Cancer classification for Merkel cell carcinoma and histopathological parameters that determine prognosis. Journal of Clinical Pathology. $2019 \quad 01 \quad 13 ; \quad$ ID $\quad$ jclinpath-2018205504.https://doi.org/10.1136/jclinpath-2018-205504

5. Tai $P, A u$ J. Skin cancer management-updates on Merkel cell carcinoma. Annals of Translational Medicine. 2018; 6(14): 282-282. https://doi.org/10.21037/atm.2018.06.13 
Carcinoma de células de Merkel: patogénesis, manejo y tratamientos emergentes - Dr. Daniel Esteban Barquero Orias; Dr. Denis Ulises Landaverde Recinos; Dra. Jimena María Segura Guevara

6. Schmults C, Blitzblau R, Aasi S, Alam M, Andersen J, Bordeaux J et al. Merkel Cell Carcinoma [Internet]. USA: National Comprehensive Cancer Network; 2019 [cited 12 March 2019]. Available from: https://merkelcell.org/wp-content/uploads/2017/10/MCC v.2.2019-2.pdf

7. Bishop A, Garden A, Gunn G, Rosenthal D, Beadle B, Fuller C et al. Merkel cell carcinoma of the head and neck: Favorable outcomes with radiotherapy. Head \& Neck. 2015;38(S1):E452-E458. https://doi.org/10.1002/hed.24017

8. Veness M. Radiation Therapy in Merkel Cell Carcinoma [internet]. Cham: Radiation Oncology. 2018 April 18 [cited 12 March 2019]. Available from:https://link.springer.com/referenceworkentry/10.1007/978-3-319$\underline{52619-5 \quad 16-1}$

9. Lebbe C, Becker J, Grob J, Malvehy J, del Marmol V, Pehamberger $\mathrm{H}$ et al. Diagnosis and treatment of Merkel Cell Carcinoma. European consensus-based interdisciplinary guideline. European Journal of Cancer. 2015;51(16):2396-2403. https://doi.org/10.1016/..ejca.2015.06.131

10. Becker J, Stang A, DeCaprio J, Cerroni L, Lebbé C, Veness M et al. Merkel cell carcinoma. Nature Reviews Disease Primers. 2017;3:17077. https://doi.org/10.1038/nrdp.2017.77

11. Schadendorf D, Lebbé $C$, zur Hausen A, Avril M, Hariharan S, Bharmal M et al. Merkel cell carcinoma: Epidemiology, prognosis, therapy and unmet medical needs. European Journal of Cancer. 2017;71:5369. https://doi.org/10.1016/i.ejca.2016.10.022

12. Riethdorf S, Hildebrandt L, Heinzerling L, Heitzer E, Fischer N, Bergmann S et al. Detection and Characterization of Circulating Tumor Cells in Patients with Merkel Cell Carcinoma. Clinical Chemistry. 2019;65(3):462-472. https://doi.org/10.1373/clinchem.2018.297028

13. Clarke C, Robbins H, Tatalovich Z, Lynch C, Pawlish K, Finch J et al. Risk of Merkel Cell Carcinoma After Solid Organ Transplantation. JNCl Journal of the National Cancer Institute. 2015;107(2):dju382dju382. https://doi.org/10.1093/inci/dju382

14. Harms P. Update on Merkel Cell Carcinoma. Clinics in Laboratory Medicine. 2017;37(3):485501.https://doi.org/10.1016/i.cll.2017.05.004

15. Sahi H, Sihto H, Artama M, Koljonen V, Böhling T, Pukkala E. History of chronic inflammatory disorders increases the risk of Merkel cell carcinoma, but does not correlate with Merkel cell polyomavirus infection. British Journal of Cancer. 2016;116(2):260-264.https://doi.org/10.1038/bic.2016.391

16. Liu W, MacDonald M, You J. Merkel cell polyomavirus infection and Merkel cell carcinoma. Current Opinion in Virology. 2016;20:20-27. https://doi.org/10.1016/i.coviro.2016.07.011

17. Shirley M. Avelumab: A Review in Metastatic Merkel Cell Carcinoma. Targeted Oncology. 2018;13(3):409416. https://doi.org/10.1007/s11523-018-0571-4

18. Harms $\mathrm{P}$, Harms K, Moore $\mathrm{P}$, DeCaprio J, Nghiem $\mathrm{P}$, Wong $\mathrm{M}$ et al. The biology and treatment of Merkel cell carcinoma: current understanding and research priorities. Nature Reviews Clinical Oncology. 2018;15(12):763-776. https://doi.org/10.1038/s41571-018-0103-2

19. Wong S, Waldeck K, Vergara I, Schroder J, Madore J, Wilmott J et al. UV-Associated Mutations Underlie the Etiology of MCV-Negative Merkel Cell Carcinomas. Cancer Research. 2015;75(24):52285234. https://doi.org/10.1158/0008-5472.CAN-15-1877

20. Veija T, Sarhadi V, Koljonen V, Bohling T, Knuutila S. Hotspot mutations in polyomavirus positive and negative Merkel cell carcinomas. Cancer Genetics. 2016;209(1-2):3035.https://doi.org/10.1016/i.cancergen.2015.11.006 
Carcinoma de células de Merkel: patogénesis, manejo y tratamientos emergentes - Dr. Daniel Esteban Barquero Orias;

Dr. Denis Ulises Landaverde Recinos; Dra. Jimena María Segura Guevara

21. Chteinberg E, Sauer C, Rennspiess D, Beumers L, Schiffelers L, Eben J et al. Neuroendocrine Key Regulator Gene Expression in Merkel Cell Carcinoma. Neoplasia. 2018;20(12):12271235.https://doi.org/10.1016/i.neo.2018.10.003

22. Prieto I, de la Fuente $\mathrm{T}$, Medina $\mathrm{S}$, Castelo B, Sobrino B, Fortes $\mathrm{J}$ et al. Merkel cell carcinoma: An algorithm for multidisciplinary management and decision-making. Critical Reviews in Oncology/Hematology. 2016;98:170-179. https://doi.org/10.1016/j.critrevonc.2015.10.008

23. Cassler N, Merrill D, Bichakjian C, Brownell I. Merkel Cell Carcinoma Therapeutic Update. Current Treatment Options in Oncology. 2016;17(7):36. https://doi.org/10.1007/s11864-016-0409-1

24. Kline L, Coldiron B. Mohs Micrographic Surgery for the Treatment of Merkel Cell Carcinoma. Dermatologic Surgery. 2016;42(8):945-951. https://doi.org/10.1097/DSS.0000000000000801

25. Strom T, Carr M, Zager J, Naghavi A, Smith F, Cruse C et al. Radiation Therapy is Associated with Improved Outcomes in Merkel Cell Carcinoma. Annals of Surgical Oncology. 2016;23(11):35723578. https://doi.org/10.1245/s10434-016-5293-1

26. Mendenhall W, Morris C, Kirwan J, Amdur R, Shaw C, Dziegielewski P. Management of cutaneous Merkel cell carcinoma. Acta Oncologica. 2017;57(3):320-323.https://doi.org/10.1080/0284186X.2017.1349926

27. Chan I, Bhatia S, Kaufman H, Lipson E. Immunotherapy for Merkel cell carcinoma: a turning point in patient care. Journal for ImmunoTherapy of Cancer. 2018;6(1):23.https://doi.org/10.1186/s40425-018-0335-9

28. Hauschild A, Schadendorf D. Checkpoint inhibitors: a new standard of care for advanced Merkel cell carcinoma?. The Lancet Oncology. 2016;17(10):1337-1339. https://doi.org/10.1016/S1470-2045(16)30441$\underline{7}$

29. Colunga A, Pulliam T, Nghiem P. Merkel Cell Carcinoma in the Age of Immunotherapy: Facts and Hopes. Clinical Cancer Research. 2017;24(9):2035-2043. https://doi.org/10.1158/1078-0432.CCR-17-0439

30. Galluzzi L, Kroemer G. Novel immune checkpoint blocker to treat Merkel cell carcinoma. Oncolmmunology. 2017;6(6):e1315496. https://doi.org/10.1080/2162402X.2017.1315496 